\title{
A Estratégia de Pedir Ajuda em Estudantes do Ensino Fundamental
}

Help-Seeking Strategy

Among Elementary School Students

El Uso de la Estrategia de Pedir Ayuda en los Estudiantes de la Escuela Primaria

Tania Maria Serafim Rede Estadual de Ensino do Estado de São Paulo

Evely Boruchovitch Universidade Estadual de Campinas
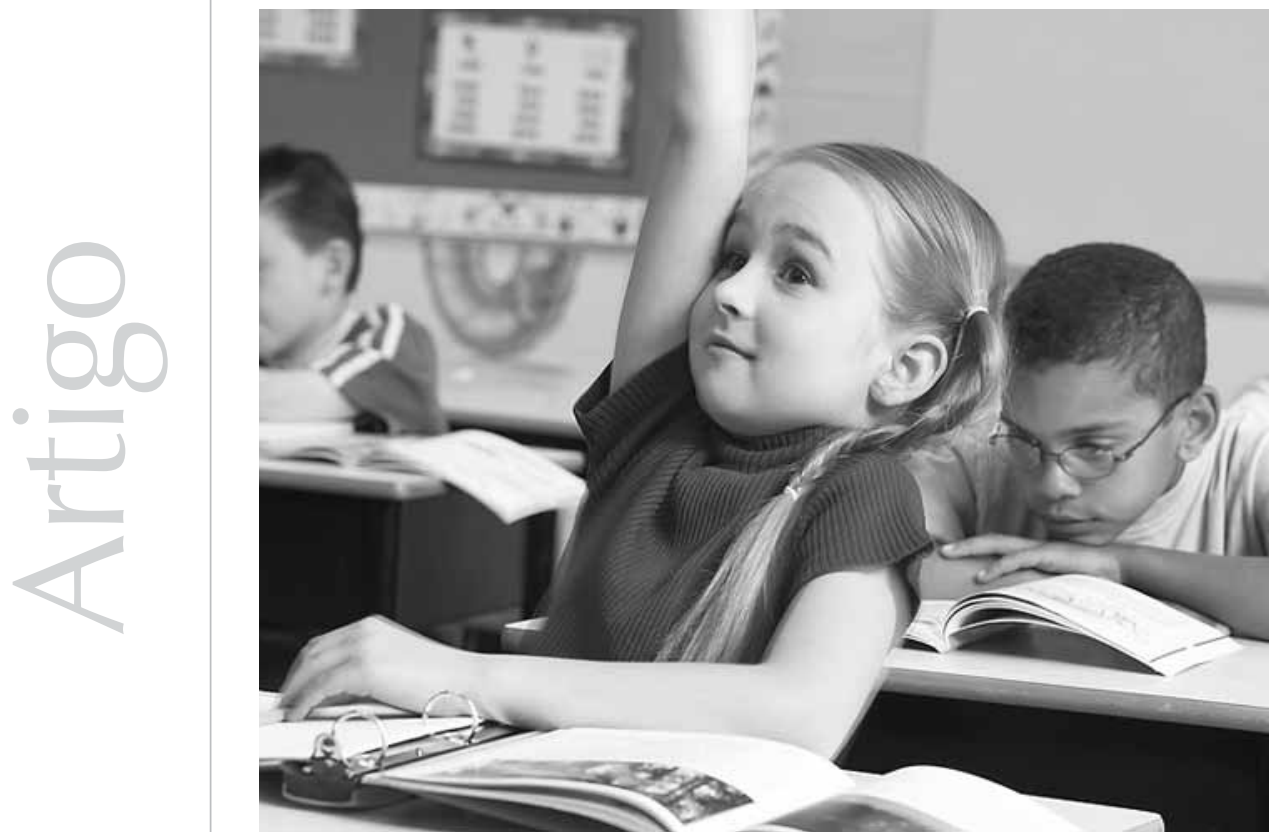
Resumo: O presente estudo objetivou verificar a relação entre a estratégia de pedir ajuda e as variáveis série escolar e gênero. Participaram da pesquisa 159 estudantes de ambos os sexos, de 2a a 4 $^{\mathrm{a}}$ séries do ensino fundamental. Os dados foram coletados por meio da Escala de Avaliação da Estratégia de Pedir Ajuda no Contexto Escolar, e foram analisados quantitativamente. Os resultados indicam que a variável série escolar parece estar relacionada ao uso do pedir ajuda. Estudantes da $2^{2} \underline{a}$ série, diferentemente dos da 3ํㅡ e 4ํㅗ séries, relataram não utilizar muito essa estratégia. Em relação ao gênero, parece que a diferença, no que diz respeito ao uso do pedir ajuda, se mostra pequena. Os dados são discutidos em termos da possibilidade de o professor favorecer o uso da estratégia de pedir ajuda no contexto de sala de aula bem como da importância dessa estratégia para a promoção da aprendizagem autorregulada.

Palavras-chave: Psicologia cognitiva. Ajuda. Aprendizagem. Ensino fundamental.

Abstract: The objective of the present study was to examine the academic help-seeking strategy in relation to school grade level and gender. The sample consisted of 159 students of both sexes, from 2nd to 4th grades. Data were collected through a Help Seeking in School Scale and analyzed quantitatively. Results showed that the school grade level was associated with help-seeking behavior. Second grade level students were the ones who reported to seek less help when compared to their third and fourth grade counterparts. Very few gender differences emerged. Data is discussed not only in terms of the importance that teachers promote this strategy use in the classroom, but also in terms of its relevance for self-regulated learning.

Keywords: Cognitive psychology. Help-seeking. Learning. Basic education.

Resumen: El objetivo de este estudio fue examinar la estratégia de pedir ayuda em relación con el grado escolar y sexo. La muestra fue de 159 estudiantes de ambos sexos del $2^{\underline{o}}$ al $4^{\mathbf{o}}$ grado. Los datos fueron recolectados través de uma escala de pedir ayuda em la sala de clase, siendo analizados cuantitativamente. Los resultados indican que el grado escolar parece relacionarse con el uso de pedir auda. Los estudiantes de $2^{\mathrm{o}}$ grado, diferente de los del $3^{\mathrm{o}}$ y $4^{\mathrm{o}}$ grado respondieron no utilizar mucho esta estrategia. Em relación al gênero, se observa uma diferencia pequeña. Los datos son discutidos em términos de favorecer el uso de la estrategia de pedir ayuda em clases, así como, también la importancia de esa estratégia para la autoregulación del aprendizaje.

Palavras clave: Psicología cognitiva. Ayuda, Aprendizaje. Educación básica.

Estudos recentes confirmam o uso das estratégias de aprendizagem como mediadoras de um bom desempenho escolar (Almeida, 2002; Boruchovitch, 1998; Costa, 2000). A Psicologia cognitiva, baseada na Teoria do Processamento da Informação, considera que a prática pedagógica deve focalizar o ensino das estratégias de aprendizagem cognitivas e metacognitivas inserindo-as no processo de aprendizagem, para favorecer o processo de autorregulação dos estudantes desde o início da escolarização formal (Boruchovitch, 2004; Dembo, 2000; Pozo, 1996; Woolfolk, 2000).
A estratégia de pedir ajuda é muito importante por ser uma estratégia metacognitiva e auto-regulatória (Ellis, 1997; Karabenick \& Sharma, 1994; Newman, 1994, 1998; Zimmerman \& Martinez-Pons, 1988). Newman (1998) afirma que pedir ajuda pode ser considerada uma estratégia metacognitiva por causa de sua natureza reflexiva, isto é, porque os estudantes refletem sobre suas próprias atividades mentais, especialmente nos momentos de dificuldade. É qualitativamente diferente de outras estratégias utilizadas pelos alunos, visto que ela é, por definição, uma transação social que envolve professores, familiares 
e pares. Assim, pedir ajuda acadêmica necessariamente abarca outras pessoas com as quais o aluno terá que interagir e de quem possivelmente receberá assistência. Nesse sentido, pedir ajuda não deve ser visto apenas como estratégia no repertório dos alunos, mas também como fator que promove um processo sociointeracional em sala de aula (Newman, 1990).

De acordo com Nelson-Le Gall (1981, 1985), há duas formas de pedir ajuda: a instrumental e a executiva. Pedir explicação indica o desejo do aluno de esclarecer ou refinar suas ideias sobre um determinado conhecimento e revela, sobretudo, a vontade de aprender, caracterizando, assim, um pedido do tipo instrumental, também chamado de adaptativo. Por sua vez, pedir a resposta correta caracteriza o tipo de pedido de ajuda executivo, pois, além de mostrar falta de conhecimento por parte do aluno, revela que ele não tem por objetivo aprender, mas sim, finalizar a tarefa.

Constatam-se na literatura internacional vastas pesquisas acerca da estratégia de pedir ajuda nos diferentes níveis de ensino. Já a literatura nacional é escassa. Mais precisamente, pode-se dizer que não foram encontradas pesquisas que abordassem especificamente a estratégia de pedir ajuda no repertório dos estudantes, em qualquer nível de ensino. No entanto, cabe salientar que, em estudos nacionais voltados para a identificação das estratégias de aprendizagem de alunos brasileiros, pedir ajuda emerge como uma das principais e mais frequentes estratégias relatadas pelos alunos para lidarem com situações de estudo e aprendizagem (Boruchovitch
1995, 1998, 1999, 2006; Costa, 2000; Schilieper, 2001; Serafim, 2004).

Nas pesquisas realizadas por Costa (2000) e Schieliper (2001), alunos de séries iniciais relataram mais o uso da estratégia de pedir ajuda do que os alunos nas séries escolares mais avançadas. No estudo de Serafim (2004), o pedir ajuda foi mencionado por alunos tanto da $2^{\underline{a}}$ série quanto da $4^{\mathrm{a}}$. Nesses estudos, a estratégia de pedir ajuda apareceu principalmente em situações de aprendizagem em sala de aula, para redigir uma redação, para lidar com a compreensão, para realizar a lição de casa ou para se preparar e realizar uma prova. Já no estudo de Boruchovitch (2006a), a estratégia de pedir ajuda foi a mais mencionada pelos alunos, e emergiu em 12 das 15 perguntas feitas na entrevista.

Pode-se constatar que diversas variáveis se mostram relacionadas tanto a pedir assistência acadêmica quanto a sua evitação. A idade, a série, o gênero, a motivação e a crença de autoeficácia constituem o conjunto dos principais fatores associados ao fato de o aluno pedir ajuda (Newman, 1993, 2000; Ryan \& Pintrich, 1997; Ryan, Gheen, \& Midgley, 1998).

Newman (2000) observou que há uma associação positiva entre a idade e o pedir ajuda adaptativo. Estudos têm revelado que os estudantes da 4a série mostraram um pedir ajuda mais adaptativo do que os das séries iniciais (Nelson-Le Gall \& Jones, 1990; Newman \& Schwager, 1995). Crianças pequenas já são capazes de diferenciar qual a melhor pessoa para auxiliá-las em suas necessidades, e essa habilidade aumenta com o avançar da 
idade e da escolarização (Newman, 2000, 2002). Os alunos de pré-escola e da primeira série do ensino fundamental julgam que uma determinada pessoa é boa em ajudar se ela foi útil em algum momento anterior (Newman, 2006). Por sua vez, os estudantes das demais séries do ensino fundamental consideram seus professores bons fornecedores de ajuda, dependendo da sua disponibilidade e da dedicação que Ihes é dada bem como da relação pessoal estabelecida entre professor e aluno.

Apesar de os alunos adquirirem melhores habilidades para pedir ajuda e de ocorrer melhor utilização dessa estratégia à medida que ficam mais velhos, há também um aumento considerável do evitar pedir ajuda com o avançar do processo de escolarização (Good et al., 1987, Newman, 1990). Não se sabe se as diferenças relacionadas à série refletem mudança no desenvolvimento cognitivo-social ou mudanças no contexto de sala de aula. Trata-se, pois, de uma questão complexa e que, segundo alguns autores, demanda pesquisas mais aprofundadas (Newman \& Schwager, 1995).

Pesquisas também não são conclusivas quanto à diferença de gênero no que concerne ao pedir ajuda. Newman e Goldin (1990) verificaram que as meninas demonstram maior preocupação em não parecer burras, quando fazem perguntas ao professor em aula de matemática. Em um outro trabalho, Newman e Schwager verificaram que as meninas da 5 a série demonstravam ser mais preocupadas do que os meninos em fazer perguntas ao professor.
Boruchovitch (1999) menciona ser necessário o desenvolvimento de mais pesquisas sistemáticas que objetivem a ampliação do conhecimento sobre o uso das estratégias de aprendizagem por parte dos estudantes brasileiros. Segundo a autora, o estudo dessa temática tem constituído uma nova área de investigação para os profissionais que trabalham com aprendizagem humana.

Assim, considerando a relevância das estratégias de aprendizagem para o êxito acadêmico, a importância da estratégia de pedir ajuda como estratégia metacognitiva para a aprendizagem auto-regulada e a necessidade de maior conhecimento acerca do uso das estratégias por parte dos estudantes, o presente estudo relata os resultados de uma pesquisa descritiva e correlacional que objetivou examinar a relação entre a estratégia de pedir ajuda e as variáveis série escolar e idade entre alunos do ensino fundamental.

\section{Método}

\section{Participantes}

Fizeram parte do estudo 159 estudantes de ambos os sexos, sendo 59 alunos de $2^{a}$ série $(37,1 \%), 58$ estudantes de 3 a série $(37,1 \%)$ e 42 alunos de 4 a série $(28,5 \%)$ de uma escola pública do ensino fundamental de Campinas. Dos participantes, $46 \%$ eram meninos e $54 \%$, meninas. A faixa etária variou de 7 a 11 anos, sendo a média de idade 9 anos. A pesquisa envolveu 4 salas de aula de $2^{\underline{a}}$ série, 3 , de $3^{\text {a }}$ série e 3 , de 4 a série, totalizando 10 salas. Cada sala de aula conta com um professor multidisciplinar, um especialista em artes e outro em educação física. 
Instrumento

\section{Escala de Avaliação da Estratégia de Pedir Ajuda no Contexto Escolar (EAPA-EF)}

Os dados relativos à estratégia de pedir ajuda foram coletados por meio da escala The Mathematics Learning in the Classroom Questionnaire (Newman, 1990), traduzida e adaptada por Serafim e Boruchovitch (2007). Inicialmente, o autor foi contatado e autorizou o uso, a tradução e a adaptação do instrumento ao contexto brasileiro. $\mathrm{O}$ instrumento original apresenta o formato de escala Likert, e consta de quatro subescalas em relação ao pedir ajuda à aprendizagem da matemática: percepção do ambiente de sala de aula (9 itens), estratégias de sala de aula (18 itens), atitudes e crenças sobre pedir ajuda (10 itens) e atitudes, crenças e metas de realização (13). Cada subescala possui instruções próprias relativas ao seu conteúdo.

Como o material é de língua inglesa, primeiramente foi realizada a tradução do inglês para o português por duas pessoas isoladamente. A concordância na tradução dos itens foi estimada, e ficou em torno de $85 \%$. As poucas discordâncias foram resolvidas por consenso, prevalecendo a melhor tradução do item para a sua aplicação no contexto brasileiro. Em seguida, foi solicitado a um estudante norte-americano que fizesse a versão do instrumento para o inglês (back translation). A concordância na tradução dos itens foi estimada, e ficou em torno de $90 \%$. Acredita-se que os cuidados metodológicos tomados, no que concerne à tradução e à adaptação do instrumento, possibilitaram a mensuração da variável estudada de maneira válida e confiável.

Após o processo de tradução, foi consenso entre as pesquisadoras adequar as questões de modo que fosse avaliado o pedir ajuda em situações variadas de aprendizagem escolar, e não só em situações relativas à matemática, como propunha o instrumento original. Houve concordância também em adotar as alternativas sempre, às vezes e nunca, ao invés de cinco alternativas de respostas, de 1 (totalmente falso) até 5 (totalmente verdadeiro), visto que a experiência prévia em avaliação psicoeducacional tem revelado que os alunos das séries iniciais do ensino fundamental apresentam dificuldades com instrumentos com muitas opções de respostas, sendo essas três as mais sensíveis a crianças (Boruchovitch, 2006b).

Assim, a escala traduzida e adaptada foi composta de 43 itens divididos entre 4 subescalas, que buscaram investigar a estratégia de pedir ajuda em relação à percepção do ambiente de sala de aula e o pedir ajuda (8 itens), às estratégias de sala de aula e o pedir ajuda (9 itens), às situações nas quais se pede ajuda (12 itens), às atitudes e às crenças sobre pedir ajuda (14 itens). O alpha de Cronbach da escala total é 0,60 , na presente amostra, revelando que o instrumento apresenta bom índice de consistência interna (Prieto \& Muniz, 2000). Vale ressaltar que, no presente estudo, serão apresentados os resultados relativos ao escore total dos participantes, e não às subescalas. 


\section{Procedimentos}

Inicialmente, o projeto de pesquisa foi submetido à avaliação do Comitê de Ética da Faculdade de Ciências Médicas da Unicamp, a fim de que fosse realizada dentro dos princípios éticos estabelecidos por esse órgão competente, tendo sido aprovado (Processo no 754/2007).

Posteriormente, foi realizado um contato com a escola durante o qual foram esclarecidos os objetivos da pesquisa bem como sua importância para a produção e o avanço do conhecimento acadêmico e da prática pedagógica. Foi garantido à direção da escola que esta não teria seu nome mencionado, e que a coleta de dados com alunos e professores seria realizada nos horários de maior conveniência da instituição. Foi enviado aos pais um termo de consentimento esclarecendo a forma de participação dos estudantes.

Após estabelecer um bom rapport com os participantes, o instrumento foi administrado em grupos de 10 alunos. A pesquisadora fez a leitura em voz alta de todos os itens e acompanhou o seu preenchimento. A coleta de dados teve duração de 40 a 50 minutos, variando em função das séries e da idade dos participantes.

\section{Análise de dados}

A análise dos dados foi quantitativa, com base nos procedimentos de estatística descritiva e inferencial. Foi utilizada a técnica de análise de cluster a fim de agrupar os participantes com concepções semelhantes em relação ao uso da estratégia de pedir ajuda. Para a variável série escolar, foi utilizado o Teste de Kruskal-Wallis para identificação dos itens nos quais ocorrem diferenças significativas, seguido do MannWhitney sobre os itens que apresentaram significância estatística para analisar e comparar o perfil de cada série escolar. $\mathrm{O}$ teste não paramétrico de Mann-Whitney foi empregado para verificar se havia diferenças estatisticamente significativas entre a estratégia de pedir ajuda e a variável gênero.

\section{Resultados}

De acordo com os testes estatísticos realizados, os 159 participantes foram agrupados em 2 clusters distintos. O primeiro é formado por 107 sujeitos, e o segundo, por 52.

Inicialmente, serão apresentados os dados relativos à série escolar, e, em seguida, os referentes ao gênero. Serão comentados apenas os dados que obtiveram significância estatística. O Teste Não Paramétrico, de Kruskal-Wallis, mostrou que, dos 43 itens que compõem a escala, 11 são estatisticamente significativos. O Teste de Mann-Whitney foi neles aplicado para analisar e comparar o perfil de cada série nesses itens específicos.

A Tabela 1 apresenta a comparação entre a $2 \underline{a}$ e a $3 \underline{a}$ séries, e indica que essas séries diferem em seis assertivas. Já a Tabela 2 mostra detalhadamente as opiniões dos participantes em relação aos itens que apresentam significância estatística. 
Tabela 1. Teste de Mann-Whitney: comparação entre as respostas dos participantes da $2^{2} \underline{a}$ e da $3^{a}$ séries para a Escala de Avaliação da Estratégia de Pedir Ajuda no Contexto Escolar.

\begin{tabular}{|c|c|c|c|}
\hline Assertivas & $\begin{array}{c}\text { Mann- } \\
\text { Whitney U }\end{array}$ & $\mathbf{Z}$ & Significância \\
\hline $\begin{array}{l}\text { A professora esta sempre muito ocupada para responder } \\
\text { as perguntas dos alunos. }\end{array}$ & 1509,000 & $-1,446$ & 148 \\
\hline A professora gosta de responder as perguntas dos alunos. & 1560,500 & $-1,086$ & 277 \\
\hline $\begin{array}{l}\text { Quando você está fazendo sua tarefa na sala de aula, } \\
\text { você pode fazer perguntas para a professora. }\end{array}$ & 1470,000 & $-1,554$ & 120 \\
\hline $\begin{array}{l}\text { Se tem uma tarefa que eu não consigo fazer, eu pulo e } \\
\text { vou para a próxima sem pedir ajuda. }\end{array}$ & 972,500 & $-4,572$ &, $000^{* * *}$ \\
\hline Quando eu preciso de ajuda, eu peço para outro aluno. & 1224,500 & $-2,997$ &, $003^{* *}$ \\
\hline $\begin{array}{l}\text { Eu não consigo me lembrar de alguma coisa que eu } \\
\text { preciso saber para fazer a tarefa. }\end{array}$ & 1338,500 & $-2,379$ &, $017^{*}$ \\
\hline Eu estou estudando uma matéria nova e sinto dificuldade. & 1415,500 & $-1,885$ & ,059 \\
\hline $\begin{array}{l}\text { Eu preciso de ajuda com alguma coisa que a professora já } \\
\text { explicou como fazer. }\end{array}$ & 1205,000 & $-3,259$ &, $001^{* * *}$ \\
\hline $\begin{array}{l}\text { Eu acho que fazer perguntas à professora me ajuda a } \\
\text { aprender as matérias. }\end{array}$ & 1384,000 & $-2,061$ &, $039 *$ \\
\hline Eu tenho vergonha de fazer perguntas. & 1197,500 & $-3,312$ &, $001^{* * *}$ \\
\hline $\begin{array}{l}\text { Eu acho que as outras crianças da classe podem pensar } \\
\text { que eu sou burro quando eu faço perguntas. }\end{array}$ & 1530,500 & $-1,408$ & ,159 \\
\hline
\end{tabular}

Nota: * Significante a 0,05, ** Significante a 0,01 e *** Significante a 0,001

Tabela 2. Comparação de respostas mencionadas pelos participantes da $2^{2} \underline{a}$ e da $3^{\underline{a}}$ séries.

\begin{tabular}{|c|c|c|c|c|c|c|}
\hline & & $2^{\mathbf{a}}$ série & & & $3^{\mathbf{a}}$ série & \\
\hline \multirow{3}{*}{ Assertivas } & Sempre & Às vezes & Nunca & Sempre & Às vezes & Nunca \\
\hline & $\mathbf{N}$ & $\mathbf{N}$ & $\mathbf{N}$ & $\mathbf{N}$ & $\mathbf{N}$ & $\mathbf{N}$ \\
\hline & $\%$ & $\%$ & $\%$ & $\%$ & $\%$ & $\%$ \\
\hline \multirow{2}{*}{$\begin{array}{l}\text { Se tem uma tarefa que eu não consigo fazer, } \\
\text { eu pulo e vou para a próxima sem pedir } \\
\text { ajuda. }\end{array}$} & 7 & 7 & 45 & 9 & 34 & 16 \\
\hline & 11,9 & 11,9 & 76,3 & 15,3 & 57,6 & 27,1 \\
\hline \multirow{2}{*}{$\begin{array}{l}\text { Quando eu preciso de ajuda, eu peço } \\
\text { para outro aluno. }\end{array}$} & 8 & 16 & 35 & 17 & 23 & 19 \\
\hline & 13,6 & 27,1 & 59,3 & 28,8 & 39,0 & 32,2 \\
\hline \multirow{2}{*}{$\begin{array}{l}\text { Eu não consigo me lembrar de alguma coisa } \\
\text { que eu preciso saber para fazer a tarefa. }\end{array}$} & 12 & 29 & 18 & 19 & 33 & 7 \\
\hline & 20,3 & 49,2 & 30,5 & 32,2 & 55,9 & 11,9 \\
\hline \multirow{2}{*}{$\begin{array}{l}\text { Eu preciso de ajuda com alguma coisa que } \\
\text { a professora já explicou como fazer. }\end{array}$} & 3 & 14 & 42 & 10 & 24 & 24 \\
\hline & 5,1 & 23,7 & 71,2 & 16,9 & 40,7 & 40,7 \\
\hline \multirow{2}{*}{$\begin{array}{l}\text { Eu acho que fazer perguntas à professora } \\
\text { me ajuda a aprender as matérias. }\end{array}$} & 23 & 12 & 24 & 30 & 18 & 11 \\
\hline & 39,0 & 20,3 & 40,7 & 50,8 & 30,5 & 18,6 \\
\hline \multirow{2}{*}{ Eu tenho vergonha de fazer perguntas. } & 4 & 12 & 43 & 10 & 24 & 25 \\
\hline & 6,8 & 20,3 & 72,9 & 16,9 & 40,7 & 42,4 \\
\hline
\end{tabular}


A maioria dos alunos da $2^{a}$ série relatou que não pula uma lição e vai para a próxima sem pedir ajuda, e que também não tem vergonha de perguntar. Todavia, afirmaram que não pedem ajuda para outro aluno e não pedem ajuda para alguma coisa que a professora já tenha explicado. Por sua vez, a maior parte das respostas dos estudantes da $3 \underline{a}$ série se concentra na alternativa às vezes. Afirmaram que às vezes passam para a próxima tarefa sem pedir ajuda e que pedem ajuda para outros colegas. Acreditam que fazer perguntas ajuda a aprender, e revelaram timidez em fazê-las.

O resultado da comparação entre a $2^{2}$ e a $4^{\underline{a}}$ séries pode ser visto na Tabela 3. Pode-se constatar que essas séries diferem em 8 assertivas. A Tabela 4 mostra as respostas dos participantes em relação às questões que apresentaram diferenças estatisticamente significativas.

Tabela 3. Teste de Mann-Whitney: comparação entre as respostas dos participantes da $2^{\underline{a}}$ e da 4a séries para a Escala de Avaliação da Estratégia de Pedir Ajuda no Contexto Escolar (Serafim, Boruchovitch, \& Newman, 2007).

\begin{tabular}{|c|c|c|c|}
\hline Assertivas & $\begin{array}{c}\text { Mann- } \\
\text { Whitney U }\end{array}$ & $\mathbf{Z}$ & Significância \\
\hline $\begin{array}{l}\text { A professora esta sempre muito ocupada para responder } \\
\text { as perguntas dos alunos. }\end{array}$ & 854,500 & $-2,754$ &, $006^{* *}$ \\
\hline A professora gosta de responder as perguntas dos alunos. & 988,500 & $-1,763$ & ,078 \\
\hline $\begin{array}{l}\text { Quando você está fazendo sua tarefa na sala de aula, } \\
\text { você pode fazer perguntas para a professora. }\end{array}$ & 759,500 & $-3,358$ &, $001^{* * *}$ \\
\hline $\begin{array}{l}\text { Se tem uma tarefa que eu não consigo fazer, eu pulo e } \\
\text { vou para a próxima sem pedir ajuda. }\end{array}$ & 818,500 & $-3,165$ &, $002^{* *}$ \\
\hline Quando eu preciso de ajuda, eu peço para outro aluno. & 939,500 & $-2,085$ &, $037^{*}$ \\
\hline $\begin{array}{l}\text { Eu não consigo me lembrar de alguma coisa que eu } \\
\text { preciso saber para fazer a tarefa. }\end{array}$ & 1060,500 & $-1,178$ & ,239 \\
\hline Eu estou estudando uma matéria nova e sinto dificuldade. & 768,500 & $-3,338$ &, $001^{* * *}$ \\
\hline $\begin{array}{l}\text { Eu preciso de ajuda com alguma coisa que a professora já } \\
\text { explicou como fazer. }\end{array}$ & 989,500 & $-1,825$ & ,068 \\
\hline $\begin{array}{l}\text { Eu acho que fazer perguntas à professora me ajuda a } \\
\text { aprender as matérias. }\end{array}$ & 822,000 & $-2,918$ &, $004^{* *}$ \\
\hline Eu tenho vergonha de fazer perguntas. & 955,500 & $-2,105$ & ,035* \\
\hline $\begin{array}{l}\text { Eu acho que as outras crianças da classe podem pensar } \\
\text { que eu sou burro quando eu faço perguntas. }\end{array}$ & 901,500 & $-2,567$ &, $010^{*}$ \\
\hline
\end{tabular}

Nota: * Significante a 0,05, ** Significante a 0,01 e *** Significante a 0,001 
Tabela 4. Comparação de respostas mencionadas pelos participantes da $2^{2} \underline{a}$ e da $4 \underline{a}$ séries.

\begin{tabular}{|c|c|c|c|c|c|c|}
\hline & & 2a série & & & 4⿳a série & \\
\hline \multirow{3}{*}{ Assertivas } & Sempre & Às vezes & Nunca & Sempre & Às vezes & Nunca \\
\hline & $\mathbf{N}$ & $\mathbf{N}$ & $\mathbf{N}$ & $\mathbf{N}$ & $\mathbf{N}$ & $\mathbf{N}$ \\
\hline & $\%$ & $\%$ & $\%$ & $\%$ & $\%$ & $\%$ \\
\hline \multirow{2}{*}{$\begin{array}{l}\text { A professora esta sempre muito ocupada } \\
\text { para responder as perguntas dos alunos. }\end{array}$} & 20 & 33 & 6 & 7 & 21 & 13 \\
\hline & 33,9 & 55,9 & 10,2 & 17,1 & 51,2 & 31,7 \\
\hline \multirow{2}{*}{$\begin{array}{l}\text { Quando você está fazendo sua tarefa na } \\
\text { sala de aula, você pode fazer perguntas } \\
\text { para a professora. }\end{array}$} & 12 & 21 & 26 & 17 & 19 & 5 \\
\hline & 20,3 & 35,6 & 44,1 & 41,5 & 46,3 & 12,2 \\
\hline \multirow{2}{*}{$\begin{array}{l}\text { Se tem uma tarefa que eu não consigo } \\
\text { fazer, eu pulo e vou para a próxima } \\
\text { sem pedir ajuda. }\end{array}$} & 7 & 7 & 45 & 4 & 21 & 16 \\
\hline & 11,9 & 11,9 & 76,3 & 9,8 & 51,2 & 39,0 \\
\hline \multirow{2}{*}{$\begin{array}{l}\text { Quando eu preciso de ajuda, eu } \\
\text { peço para outro aluno. }\end{array}$} & 8 & 16 & 35 & 4 & 24 & 13 \\
\hline & 13,6 & 27,1 & 59,3 & 9,8 & 58,5 & 31,7 \\
\hline \multirow{2}{*}{$\begin{array}{l}\text { Eu estou estudando uma matéria } \\
\text { nova e sinto dificuldade. }\end{array}$} & 13 & 25 & 21 & 17 & 22 & 2 \\
\hline & 22,0 & 42,4 & 35,6 & 41,5 & 53,7 & 4,9 \\
\hline \multirow{2}{*}{$\begin{array}{l}\text { Eu acho que fazer perguntas à professora } \\
\text { me ajuda a aprender as matérias. }\end{array}$} & 23 & 12 & 24 & 22 & 18 & 1 \\
\hline & 39,0 & 20,3 & 40,7 & 53,7 & 43,9 & 2,4 \\
\hline \multirow{2}{*}{ Eu tenho vergonha de fazer perguntas. } & 4 & 12 & 43 & 4 & 16 & 16 \\
\hline & 6,8 & 20,3 & 72,9 & 9,8 & 39,0 & 39,0 \\
\hline \multirow{2}{*}{$\begin{array}{l}\text { Eu acho que as outras crianças da classe } \\
\text { podem pensar que eu sou burro quando } \\
\text { eu faço perguntas. }\end{array}$} & 5 & 9 & 45 & 4 & 17 & 20 \\
\hline & 8,5 & 15,3 & 76,3 & 9,8 & 41,5 & 48,8 \\
\hline
\end{tabular}

Os estudantes da $2^{2}$ relataram que não podem fazer perguntas para a professora quando estão fazendo suas lições, não pedem ajuda aos colegas quando necessitam de ajuda e não acreditam que fazer perguntas ajuda a aprender as matérias. Revelaram que não têm vergonha de fazer perguntas e que não temem o julgamento por parte dos colegas ao fazer perguntas. Por sua vez, os participantes da $4 \underline{a}$ afirmaram que às vezes pedem ajuda aos colegas e à professora, às vezes pedem ajuda com uma matéria nova e às vezes passam para a atividade seguinte sem pedir ajuda.

Os dados relativos à comparação entre a $3^{\underline{a}}$ e a $4^{\underline{a}}$ séries são apresentados na Tabela 5, que indica que essas séries diferem apenas em duas assertivas. A Tabela 6 apresenta as respostas dos participantes em relação às questões que revelaram significância estatística. 
Tabela 5. Teste de Mann-Whitney: comparação entre as respostas dos participantes da $3^{\mathfrak{a}}$ e da 4a séries para a Escala de Avaliação da Estratégia de Pedir Ajuda no Contexto Escolar.

\begin{tabular}{|c|c|c|c|}
\hline Assertivas & $\begin{array}{c}\text { Mann- } \\
\text { Whitney U }\end{array}$ & $\mathbf{Z}$ & Significância \\
\hline $\begin{array}{l}\text { A professora esta sempre muito ocupada para responder } \\
\text { as perguntas dos alunos. }\end{array}$ & 983,500 & $-1,819$ & ,069 \\
\hline A professora gosta de responder as perguntas dos alunos. & 872,500 & $-2,644$ &, $008^{* *}$ \\
\hline $\begin{array}{l}\text { Quando você está fazendo sua tarefa na sala de aula, } \\
\text { você pode fazer perguntas para a professora. }\end{array}$ & 945,500 & $-1,985$ &, $047^{*}$ \\
\hline $\begin{array}{l}\text { Se tem uma tarefa que eu não consigo fazer, eu pulo e } \\
\text { vou para a próxima sem pedir ajuda. }\end{array}$ & 1039,000 & $-1,337$ & 181 \\
\hline Quando eu preciso de ajuda, eu peço para outro aluno. & 1057,500 & $-1,153$ & 249 \\
\hline $\begin{array}{l}\text { Eu não consigo me lembrar de alguma coisa que eu } \\
\text { preciso saber para fazer a tarefa. }\end{array}$ & 1045,500 & $-1,334$ & ,182 \\
\hline Eu estou estudando uma matéria nova e sinto dificuldade. & 986,000 & $-1,736$ & ,083 \\
\hline $\begin{array}{l}\text { Eu preciso de ajuda com alguma coisa que a professora já } \\
\text { explicou como fazer. }\end{array}$ & 1019,500 & $-1,469$ & ,142 \\
\hline $\begin{array}{l}\text { Eu acho que fazer perguntas à professora me ajuda a } \\
\text { aprender as matérias. }\end{array}$ & 1085,500 &,- 965 & ,335 \\
\hline Eu tenho vergonha de fazer perguntas. & 1070,500 & $-1,065$ & ,287 \\
\hline $\begin{array}{l}\text { Eu acho que as outras crianças da classe podem pensar } \\
\text { que eu sou burro quando eu faço perguntas. }\end{array}$ & 1062,500 & $-1,169$ & ,242 \\
\hline
\end{tabular}

Nota: * Significante a $0,05, * *$ Significante a 0,01

Tabela 6. Comparação de respostas mencionadas pelos participantes da $3^{\underline{a}}$ e da $4^{\underline{a}}$ séries.

\begin{tabular}{|c|c|c|c|c|c|c|}
\hline & & $3^{\underline{a}}$ série & & & 4⿳a série & \\
\hline \multirow{3}{*}{ Assertivas } & Sempre & Às vezes & Nunca & Sempre & Às vezes & Nunca \\
\hline & $\mathbf{N}$ & $\mathbf{N}$ & $\mathbf{N}$ & $\mathbf{N}$ & $\mathbf{N}$ & $\mathbf{N}$ \\
\hline & $\%$ & $\%$ & $\%$ & $\%$ & $\%$ & $\%$ \\
\hline \multirow{2}{*}{$\begin{array}{l}\text { A professora gosta de responder as } \\
\text { perguntas dos alunos. }\end{array}$} & 21 & 31 & 7 & 24 & 17 & 0 \\
\hline & 35,6 & 52,5 & 11,9 & 58,5 & 41,5 & 0 \\
\hline \multirow{2}{*}{$\begin{array}{l}\text { Quando você está fazendo sua tarefa na sala } \\
\text { de aula, você pode fazer perguntas para a } \\
\text { professora. }\end{array}$} & 17 & 24 & 18 & 41 & 19 & 5 \\
\hline & 28,8 & 40,7 & 30,5 & 17,5 & 46,3 & 12,2 \\
\hline
\end{tabular}

Para a maioria dos estudantes da $3 \underline{\text { a }}$ série, a professora às vezes gosta de responder as perguntas dos alunos, e às vezes podem fazer perguntas para ela. Já os estudantes da 4a série mencionaram que a professora sempre gosta de responder as questões dos alunos e que sempre podem fazer perguntas enquanto estão fazendo a tarefa escolar. 
Para verificar se havia diferenças estatisticamente significativas entre a estratégia de pedir ajuda e a variável gênero, foi utilizado o Teste Não Paramétrico, de Mann-Whitney. Apenas uma assertiva apresentou significância estatística, a que dizia "Quando eu preciso de ajuda, eu peço para outro aluno". Entre os meninos, 41 nunca pedem ajuda, 22 pedem ajuda às vezes e 12 sempre pedem ajuda. Já entre as meninas, 26 nunca pedem ajuda, 41 pedem às vezes e 16 pedem sempre. $\mathrm{O}$ dado parece sugerir que as meninas se mostram mais propensas a pedir ajuda aos colegas, quando comparadas aos meninos.

\section{Discussão}

No que se refere à série escolar, os dados indicam que a $2^{2}$ série é a que mais se distingue das demais séries em relação ao uso da estratégia de pedir ajuda, pois se diferencia da $3^{\underline{a}}$ série em 6 assertivas e da $4 \underline{a}$ série em 8 itens. Já a $3^{\underline{a}}$ e a $4 \underline{a}$ séries apresentaram poucas diferenças estatisticamente significativas entre si, apenas 2 assertivas. Os dados sugerem que os alunos da 2ạ série têm uma percepção mais negativa do pedir ajuda quando comparados com os estudantes da $3^{\underline{a}}$ e da $4^{\underline{a}}$ séries. Os estudantes da 2 a série mencionaram que não pedem ajuda para o colega da classe, não costumam pedir ajuda para a professora depois que ela explicou como fazer, consideram que não podem pedir ajuda para a professora quando estão fazendo a tarefa e não acreditam que fazer perguntas os ajude a aprender. Já os estudantes da $3^{\underline{a}}$ e $4 \underline{a}$ relataram pedir ajuda para os colegas e acreditar que fazer perguntas os ajuda a aprender as matérias.

Essa diferença entre as séries no que concerne à estratégia pedir ajuda encontrada no presente estudo foi instigante. É difícil precisar se foi uma característica da amostra investigada ou da escola onde a pesquisa se realizou, ou se ela se deve a outros fatores relativos à escolarização, de modo geral. Considerando as importantes implicações educacionais desse dado, já que educadores podem atuar, desde o início da escolarização, no sentido de criarem ambientes em sala de aula que promovam mais ou menos a aprendizagem, recomenda-se que as relações entre a série escolar e o comportamento de pedir ajuda em sala de aula sejam mais exaustivamente examinadas pela pesquisa futura.

Segundo Newman (2000, 2002, 2006), as pesquisas têm indicado que crianças pequenas já são capazes de escolher as pessoas ideais para ajudá-las, e que essa habilidade aumenta com a idade. Alunos da pré-escola e da 1á série do ensino fundamental acreditam que uma pessoa é boa em ajudar se ela já foi útil no passado.

A relação entre professor e aluno é fundamental no uso da estratégia de pedir ajuda. No estudo realizado por Newman e Goldin (1990), os alunos das séries iniciais do ensino fundamental revelaram temer reações negativas por parte do professor ao pedir ajuda. O custo de pedir ajuda ao professor é reforçado quando ele não demonstra desejo em ajudar. Comentários tais como "Se você tivesse prestado atenção, agora não precisaria de ajuda" desencorajam qualquer iniciativa de utilizar a estratégia de pedir ajuda.

No presente estudo, o gênero parece não estar associado a diferenças no uso da estratégia de pedir ajuda, uma vez que apenas um item revelou significância estatística. Resultados de um conjunto de pesquisas têm indicado que o 
gênero não aparece como variável significativa em relação à estratégia de pedir ajuda (NelsonLe Gall \& Glor-Scheib, 1985; Nelson-Le Gall \& Jones, 1990; Newman, 1990; Van der Meij, 1988, 1990) ao passo que outros estudos têm apresentado pequenas diferenças com relação ao gênero (Arbretron, 1998; Newman \& Schwager, 1993). Segundo Arbretron 1998, essas diferenças se mostram muito mais relacionadas a padrões motivacionais distintos entre meninos e meninas do que propriamente a quem utiliza mais a estratégia de pedir ajuda. Pesquisas voltadas para a relação entre a estratégia de pedir ajuda e o gênero precisam ser conduzidas, a fim de se avaliar mais claramente a relação dessa variável sobre o comportamento de pedir ajuda.

Cabe mencionar que o presente estudo constituiu uma primeira tentativa de conhecer melhor as estratégias de pedir ajuda que os estudantes utilizam no contexto educacional. Ao empregar um instrumento de autorrelato, apresenta uma limitação, pois não foi possível precisar se, de fato, os alunos que fizeram parte da amostra utilizam tal estratégia e como a utilizam. Futuros estudos devem ser conduzidos com base na observação do comportamento dos estudantes em sala de aula. Uma outra limitação do presente estudo concerne ao fato de a literatura mostrar que existem dois tipos de pedir ajuda: o executivo e o instrumental, formas essas com importantes e diferentes implicações para a aprendizagem. Nesse sentido, a escala utilizada na presente pesquisa não foi sensível a essa diferenciação. Assim, espera-se que as limitações ora identificadas motivem novas investigações.

\section{Considerações finais}

Pozo (1998) considera que é inegável a importância das estratégias da aprendizagem para a educação, na medida em que são instrumentos para a aquisição do conhecimento e para melhor êxito acadêmico. Sua utilização eficaz depende de um de conhecimento sobre elas, de modo a avaliar a quais estratégias recorrer para estudar determinado material. A estratégia de pedir ajuda é importante, pois envolve habilidades metacognitivas e autorreguladoras, uma vez que contribui para alertar o aluno quando algo não foi compreendido. Trata-se de uma percepção acerca do próprio processo de aprendizagem.

Compete ao professor estar atento aos seus alunos e incentivá-los a pedir ajuda nos momentos de dificuldade desde o início do processo de escolarização formal, mostrandoIhes que a dúvida e a dificuldade fazem parte do processo de aprendizagem e que utilizar a estratégia de pedir ajuda pode ser uma forma eficaz de resolvê-las. Além disso, é imprescindível o professor criar um ambiente em sala de aula que promova a aprendizagem, que acolha a dificuldade do aluno e the dê autonomia, pois a aprendizagem requer papel ativo dos alunos. 


\section{Tania Maria Serafim*}

Pedagoga e Mestre em Psicologia, Desenvolvimento Humano e Educação pela Faculdade de Educação da Unicamp, professora efetiva da Rede Estadual de Ensino, São Paulo, SP - Brasil.

E-mail: taniaserafim@yahoo.com

\section{Evely Boruchovitch}

Psicóloga pela Universidade do Estado do Rio de Janeiro, Ph.D em Educação pela University of Southern California, Los Angeles, EUA. Professora associada e Livre Docente do Departamento de Psicologia Educacional da Faculdade de Educação da Universidade Estadual de Campinas, Campinas, SP - Brasil.

E-mail: evely@unicamp.br

*Correspondência para o seguinte endereço:

Avenida Estados Unidos, 777, ap. 63, Jardim Nova Europa - Campinas, SP - Brasil - CEP 13040-099.

Recebido 16/6/2009, 1프 Reformulação 15/9/2009, Aprovado 30/9/2009.

\section{Referências}

Almeida, L. (1992). Facilitar a aprendizagem: ajudar os alunos a aprender e a pensar. Psicologia Escolar e Educacional, 6(2), 155-165.

Arbetron, A. (1998). Student goal orientation and help-seeking strategy use. In S.A. Karabenick (Ed.), Strategic help seeking: implication for learning and teaching (pp. 95-116). Mahwah, NJ: Lawrence Erlbaum.
Boruchovitch, E. (1995). A identificação e o estudo das variáveis associadas ao fracasso escolar brasileiro. Projeto de pesquisa realizado na qualidade de bolsista de Recém-Doutor da CNPQ. Faculdade de Educação, Departamento de Psicologia Educacional, Universidade de Estadual de Campinas, Campinas, SP. 
Boruchovitch, E. (1998, agosto). Understanding Brazilian students use of learning strategies. Resumo apresentado na 24 th. Internacional Congress of Applied Psycology, San Francisco.

Boruchovitch, E. (1999). Estratégias de aprendizagem e desempenho escolar: considerações para a prática educacional. Psicologia: Teoria e Crítica, 12(2), 361-376.

Boruchovitch, E. (2004). A auto-regulação da aprendizagem e a escolarização inicial. In E. Boruchovitch \& J. A. Bzuneck (Eds.), Aprendizagem: processos psicológicos e o contexto social na escola (pp. 55 - 88). Petrópolis, RJ: Vozes.

Boruchovitch, E. (2006a). Avaliação psicoeducacional: desenvolvimento de instrumentos à luz da psicologia cognitiva baseada na teoria do processamento da informação. Avaliação Psicológica, 5(2), 145-152.

Boruchovitch, E. (2006b). Estratégias de aprendizagem: uma análise à luz das variáveis demográficas e motivacionais. Tese de Livre Docência, Universidade Estadual de Campinas, Campinas, SP.

Costa, E. R. (2000). As estratégias de aprendizagem e ansiedade de alunos do ensino fundamental: implicações para a prática educacional. Dissertação de Mestrado, Faculdade de Educação, Universidade Estadual de Campinas, Campinas, SP.

Ellis, S. (1997). Strategy choice in sociocultural context. Developmental Review, 17(4), 490-524.

Good, T. L., Slavings, R. L., Harel, K. H., \& Emerson, H. (1987). Student passivity: A study of question-asking in K-12 classrooms. Sociology of Education, 60(1), 181-199.

Karabenick, S. A., \& Sharma, R. (1994). Seeking academic assistance as a strategic learning resource. In P. R. Pintrich, D. R. Brown \& C. E. Weinstein (Eds.), Student motivation, cognition, and learning: Essays in honor of Wilbert $J$. McKeachie (pp. 189-211). Hillsdale, NJ: Erlbaum.

Nelson-Le Gall, S. (1981). Help-seeking: An understudied problem-solving skill in children. Developmental Review, 1(3), 224-246.

Nelson-Le Gall, S. (1985). Help-seeking behavior in learning. Review of Research in Education, 12(1), 55-90.

Nelson-Le Gall, S., \& Glor-Scheid, S. (1985). Help seeking in elementary classrooms: an observational study. Contemporary Educational Psychology, 10(1), 58-71.

Nelson-Le Gall, S., \& Jones, E. (1990). Cognitive-motivational influences on the help-seeking behavior of black children. Child Development, 61, 581-589.

Newman, R. S. (1990). Children's help seeking in the classroom. The role of motivational factors and attitudes. Journal of Educational Psychology, 83(1), 71 -80,

Newman, R. S. (1994). Adaptive help seeking: A strategy of self-regulated learning. In D. H. Schunk \& B. J. Zimmerman (Eds.), Self-regulation of learning and performance: Issues and educational applications (pp. 282-301). Hillsdale, NJ: Lawrence Erlbaum.

Newman, R. S. (1998). Student's help seeking during problem solving: Influences of personal and contextual achievement goals. Journal of Educational Psychology, 90, 644 - 658.

Newman, R. S. (1998a). Adaptive help-seeking: A role of social interaction in self-regulated learning. In S. A.Karabenick (Ed.), Strategic help seeking: Implications for learning and teaching (pp. 13-37). Hillsdale, NJ: Erlbaum.

Newman, R. S. (2000). Social influences on the development of children's adaptive help seeking: The role of parents, teachers, and peers. Developmental Review, 20(3), 350404.
Newman, R. (2002). How self-regulated learners cope with academic difficulty: The role of adaptive help-seeking. Theory into Practice, 41(2), 132 -138.

Newman, R. S. (2006). Students' adaptive and nonadaptive help-seeking in the classroom: Implications for the context of peer harassment. In S. Karabenick, \& R. S. Newman (Eds.), Help seeking in academic settings: Goals, groups, and contexts (pp. 225-258). Lawrence Erlbaum.

Newman, R. S., \& Goldin, L. (1990). Children's reluctance to seek help with school work. Journal of Educational Psychology, 82(1), 92-100

Newman, R. S., \& Schwager, M. T. (1993). Students' perceptions of the teacher and classmates in relation to reported help seeking in math class. The Elementary School Journal, 94(1), 3-17

Newman, R. S., \& Schwager, M. T. (1995). Students' help seeking during problem solving: Effects of grade, goal, and prior achievement. American Educational Research Journal, 32(2), 352-37.

Pozo, J. I. (1996). Estratégias de aprendizagem. In C. Coll, J. Palácios \& A. Marchesi (Eds.), Desenvolvimento psicológico e educação: psicologia da educação (Vol. 2, pp. 176-197). Porto Alegre: Artes Médicas.

Prieto, G., \& Muñiz, J. (2000). Un modelo para evaluar la calidad de los tests utilizados en España. Recuperado em 14 de fevereiro de 2006, de http://www.cop.es/tests/ modelo.htm

Ryan, A. M., Gheen, M. H., \& Midgley, C. (1998). Why do some students avoid asking for help? An examination of the interplay among students' academic efficacy, teachers' social-emotional role, and the classroom goal structure. Journal of Educational Psychology, 90(3), 528-535.

Ryan, A. M., \& Pintrich, P. R. (1997). "Should I ask for help?" The role of motivation and attitudes in adolescents' help seeking in math class. Journal of Educational Psychology, 89(2), 329-341.

Schilieper, M. D. M. J. (2001). As estratégias de aprendizagem e as atribuições de causalidade de alunos do ensino fundamental. Dissertação de Mestrado, Faculdade de Educação, Universidade Estadual de Campinas, Campinas, SP.

Schunk, D. H., \& Zimmerman, B. J. (1994). Self-regulation of learning and performance: Issues and educational applications. Hillsdale, NJ: Lawrence Erlbaum.

Serafim, T. M. (2004). As estratégias de aprendizagem dos alunos do ensino fundamental: uma análise por gênero, série escolar e idade. Trabalho de conclusão de Curso, Faculdade de Educação, Universidade Estadual de Campinas, Campinas, SP.

Serafim, T. M. (2009). A estratégia de pedir ajuda de estudantes do ensino fundamental. Dissertação de Mestrado, Faculdade de Educação, Universidade Estadual de Campinas, Campinas, SP.

Van der Meij, H. (1988). Constrains on question asking in classrooms. Journal of Psychology Educational, 80(3), $401-405$.

Van der Meij, H. (1990). Question asking: To know that you do not know is not enough. Journal of Psychology Educational, $82(3), 505-512$.

Woolfolk, A. (2000). Psicologia da educação. Porto Alegre: Artes Médicas.

Zimmerman, B. J., \& Martinez-Pons, M. (1988). Construct validation of a strategy model of student self-regulated learning. Journal Educational Psychology, 80(3), 284-290. 